\title{
Stroke mechanisms and outcomes of isolated symptomatic basilar artery stenosis
}

Edgar A Samaniego, ${ }^{1}$ Amir Shaban, ${ }^{2}$ Santiago Ortega-Gutierrez, ${ }^{1}$ Jorge A Roa (D) , ${ }^{3}$ David M Hasan, ${ }^{4}$ Colin Derdeyn, ${ }^{5}$ Biyue Dai, ${ }^{6}$ Harold Adams, ${ }^{2}$ Enrique Leira ${ }^{2}$

To cite: Samaniego EA, Shaban A, Ortega-Gutierrez S, et al. Stroke mechanisms and outcomes of isolated symptomatic basilar artery stenosis. Stroke \& Vascular Neurology 2019;4: e000246. doi:10.1136/svn-2019-000246

Received 16 April 2019 Revised 29 May 2019 Accepted 19 June 2019 Published Online First 30 July 2019
Check for updates

(C) Author(s) (or their employer(s)) 2019. Re-use permitted under CC BY-NC. No commercial re-use. See rights and permissions. Published by BMJ.

${ }^{1}$ Neurology, Neurosurgery and Radiology, University of lowa Hospitals and Clinics, lowa City, lowa, USA

${ }^{2}$ Neurology, University of lowa Hospitals and Clinics, lowa City, lowa, USA

${ }^{3}$ Neurology and Neurosurgery, University of lowa Hospitals and Clinics, lowa City, lowa, USA ${ }^{4}$ Neurosurgery, University of lowa Hospitals and Clinics, lowa City, lowa, USA

${ }^{5}$ Radiology, University of lowa Hospitals and Clinics, lowa City, lowa, USA

${ }^{6}$ Biostatistics and Public Health, University of lowa Hospitals and Clinics, lowa City, lowa, USA

Correspondence to Dr Edgar A Samaniego; edgar-samaniego@uiowa.edu

\section{ABSTRACT}

Background While diffuse atherosclerotic disease affecting the posterior circulation has been described extensively, the prevalence, natural history and angiographic characteristics of isolated symptomatic basilar artery stenosis (ISBAS) remain unknown. Methods We reviewed our prospective institutional database to identify patients with $\geq 50 \%$ symptomatic basilar artery (BA) stenosis without significant atherosclerotic burden in the vertebral or posterior cerebral arteries. Stroke mechanism, collateral circulation, and degree and length of stenosis were analysed. The primary outcome was time from index event to new transient ischaemic attack (TIA), acute ischaemic stroke (AIS) or death. Other outcome variables included modified Rankin Scale (mRS) score on discharge and last follow-up.

Results of 6369 patients with AIS/TIA, 91 (1.43\%) had ISBAS. Seventy-three (80.2\%) patients presented with AIS and 18 (19.8\%) with TIA. Twenty-nine (31.9\%) were women and the median age was $66.8 \pm 13.6$ years. The mean follow-up time was 2.7 years. The most common stroke mechanism was artery-to-artery thromboembolism (45.2\%), followed by perforator occlusion $(28.7 \%)$ and flow-dependent/hypoperfusion (15.1\%). The percentage of stenosis was lower in patients who had favourable outcome compared with those with mRS 3-6 on discharge (78.3 \pm 14.3 vs $86.9 \pm 14.5, p=0.007$ ). Kaplan-Meier curves showed higher recurrence/death rates in patients with $\geq 80 \%$ stenosis, mid-basilar location and poor collateral circulation. Approximately $13 \%$ of patients with ISBAS presented with complete BA occlusion.

Conclusion ISBAS is an uncommon (1.43\%) cause of TIA and AIS. Men in their 60s are mostly affected, and artery-to-artery embolism is the most common stroke mechanism. Mid-basilar location, $\geq 80 \%$ stenosis and poor collateral circulation are important factors associated with worse prognosis.

\section{INTRODUCTION}

Atherosclerosis affecting intracranial and extracranial arteries is the most common stroke mechanism in the posterior circulation. The New England Medical Center Registry reported that $60 \%$ of strokes in the posterior circulation were due to atherosclerosis. ${ }^{2}$ With 407 patients included from 1988 to 1996 , this registry assessed stroke mechanism and the presence of atherosclerosis using imaging modalities that do not have the sensitivity and specificity of the current technologies and protocols.

The advent of new endovascular techniques and devices has broadened treatment options for patients with atherosclerotic disease in the posterior circulation. This has sparked the interest to better characterise the haemodynamics, presence of perforators and stroke mechanisms in the posterior circulation, specifically the basilar artery (BA).${ }^{34}$ In order to adequately study the potential of new endovascular procedures, it is necessary to better understand the natural history and stroke mechanisms of atherosclerotic lesions in different locations.

Data from the Stenting versus Aggressive Medical Therapy for Intracranial Arterial Stenosis (SAMMPRIS) trial have shown that perforator stroke is the most common complication of angioplasty and stenting of symptomatic BA atherosclerotic disease. ${ }^{5}$ It is not known whether this mechanism is inherently related to the endovascular procedure or the arterial disease itself. Since atherosclerosis is a systemic disease, patients with atherosclerosis in the posterior circulation tend to have multiple lesions, thus making assumptions of stroke mechanism challenging.

In some patients the primary atherosclerotic lesion is located in the BA. Since all previous studies have included subjects with diffuse atherosclerosis affecting also the vertebral arteries, vertebrobasilar junction and posterior cerebral arteries, the presentation and natural history of isolated symptomatic basilar artery stenosis (ISBAS) have not been fully characterised. This study describes the angiographic features and aetiological mechanisms of a cohort of patients with ISBAS. We focused on the BA given its potential for unfavourable outcomes when treated conservatively with medical therapy or invasively with endovascular procedures. 


\section{METHODS}

After obtaining institutional review board approval, we analysed our prospectively acquired database of patients who presented to our institution from 2001 to 2018 with acute ischaemic stroke (AIS) or transient ischaemic attack (TIA) due to BA atherosclerotic disease, as documented by at least $\geq 50 \%$ stenosis of the vessel lumen on CT angiography (CTA), contrast-enhanced magnetic resonance angiography (MRA), time-of-flight (TOF) sequences or digital subtraction angiography (DSA). CTA images were obtained on a 64-slice multidetector CT scan. Contrastenhanced MRA and TOF images were obtained on a 3T magnetic resonance scan.

The aim of the study was to identify patients with ISBAS. Therefore, patients with significant additional atherosclerotic involvement ( $\geq 50 \%$ stenosis) of the vertebral arteries (V1-V4 segments) or posterior cerebral arteries were excluded. The BA atherosclerotic plaque had to affect at least $\geq 50 \%$ of the vessel lumen. Patients who presented with AIS due to an acute BA occlusion were included if the underlying aetiology was the presence of an atherosclerotic plaque, seen after successful mechanical thrombectomy and as described elsewhere. ${ }^{6}$

The angiographic characteristics of the atherosclerotic lesion and imaging data were analysed independently by a neurointerventional surgeon and a vascular neurologist blinded from the clinical outcome. The level of inter-reader agreement was assessed by Cohen $\kappa$ statistics. Specific characteristics such as length of the lesion, degree of stenosis and location of the plaque were reviewed and recorded. The degree of stenosis (\%) was quantified as: $[1-(\mathrm{D}$ stenosis/D normal) $] \times 100$, where $\mathrm{D}$ stenosis is the diameter of the artery at the site of the most severe stenosis, and D normal is the diameter of the proximal normal artery. ${ }^{7}$

Lesion location was divided into three anatomical segments for analysis (figure 1): proximal (vertebrobasilar junction to the origin of the anteroinferior cerebellar arteries (AICAs)), mid-BA (AICA to the superior cerebellar arteries (SCAs)) and distal (the distal BA after the SCAs). Location and vascular distribution of the index stroke in patients who presented with AIS were also collected. Demographic characteristics, clinical presentation, stroke risk factors, treatment and outcomes were also analysed.

The stroke mechanism was categorised as either due to a perforator occlusion, flow-dependent/hypoperfusion or artery-to-artery embolism (figure 2) based on clinical and imaging data. This was similar to the criterion used for adjudication of ischaemic stroke mechanisms in the SAMMPRIS trial and described elsewhere. ${ }^{58}$ A perforator occlusion was a brainstem infarction in close proximity to the presence of a plaque in the BA that correlated with vascular territory of the perforator. The criterion for flow-dependent/hypoperfusion mechanism was an infarction and/or TIA localised distal to the BA plaque and triggered or exacerbated by orthostatism or lower blood pressure challenges. Several infarctions distal to

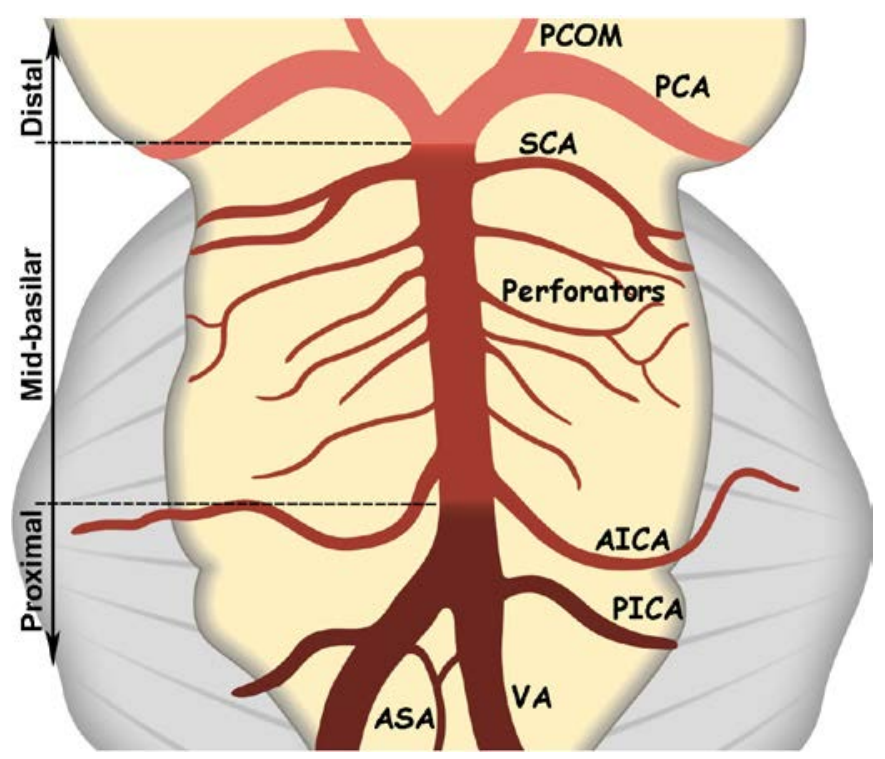

Figure 1 Basilar artery segments: proximal from the vertebrobasilar junction to the origin of the AICA, mid from the AICA to the origin of the SCA, and distal after the origin of the SCA. AICA, anteroinferior cerebellar artery; ASA, anterior spinal artery; PCA, posterior cerebral artery; PCOM, posterior communicating artery; PICA, posteroinferior cerebellar artery; SCA, superior cerebellar artery; VA, vertebral artery.

the BA plaque without evidence of orthostatism or lower blood pressure worsening were categorised as arteryto-artery thromboembolic mechanism. When more than one potential mechanism could have contributed to the stroke, the stroke mechanism was categorised as uncertain. Patients with possible cardioembolic causes of stroke, such as atrial fibrillation, mechanical valves or presence of an intracardiac thrombus on echography, were excluded from the analysis.

We assessed the degree of collateral circulation to the BA based on the presence of posterior communicating arteries (PCOMs). Whenever possible, we reviewed DSAs to determine PCOMs' patency. TOF imaging is flowdependent, and bidirectional flow through collaterals may lead to false signal loss and mischaracterisation of the vessel as being occluded. ${ }^{9}$ Conversely, contrast-enhanced CTA and MRA depict the vascular contour based on vessel patency. If the DSA was not available, CTA/MRA were preferred over TOF for BA and PCOM characterisation. Our stroke imaging protocol includes a cerebral TOF and a neck contrast-enhanced MRA that cranially extends to the circle of Willis.

A score was assigned according to the presence and diameter of PCOMs (figure 3): dominant $=2$ points; hypoplastic $=1$ point; and absent $=0$ point. The total score ranged from 0 (ie, no PCOMs) to 4 (ie, two dominant PCOMs), with $0-1$ points considered 'low/poor' collateral flow and 2-4 points 'high/good' collateral flow.

All recurrent cerebral ischaemic events (TIA/AIS/ death) after the index event were considered as the primary outcome. Secondary outcomes included functional outcome measured by the modified Rankin Scale 


\section{A.1 Perforator}
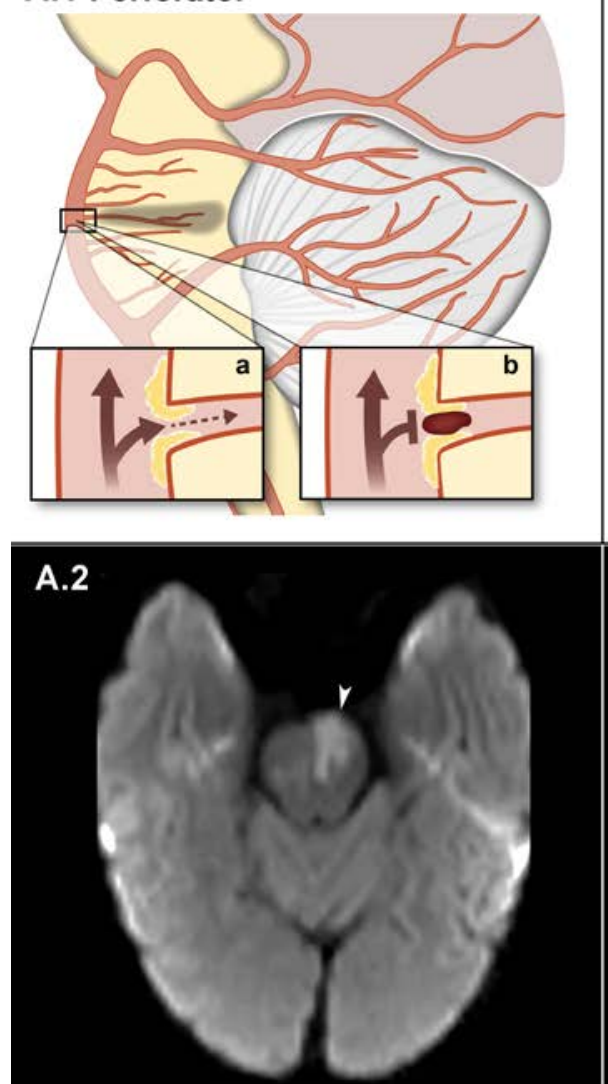

B.1 Flow-dependent
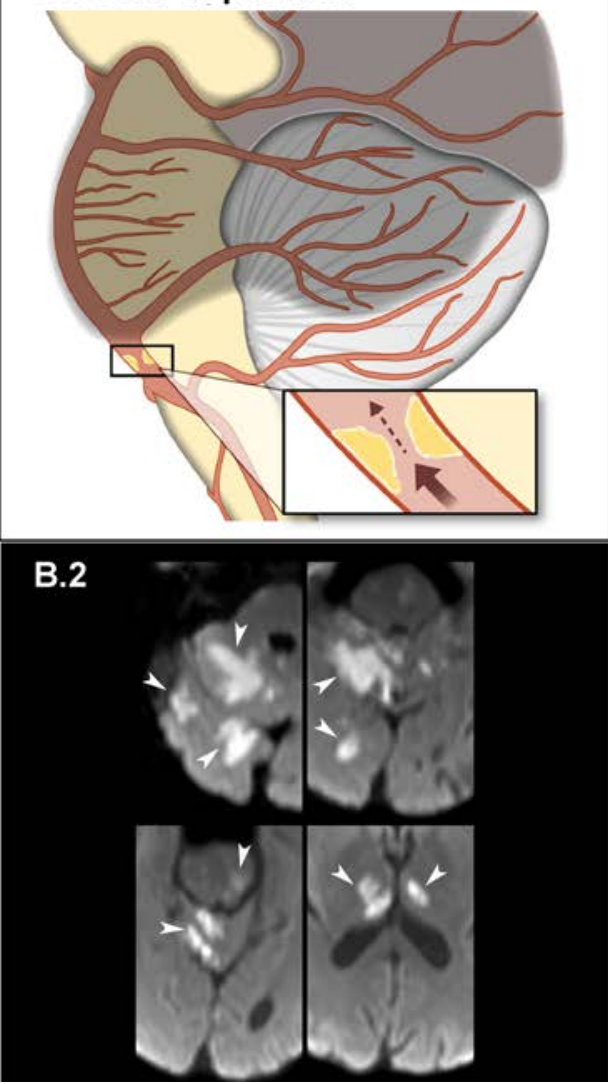

\section{C.1 Embolic}

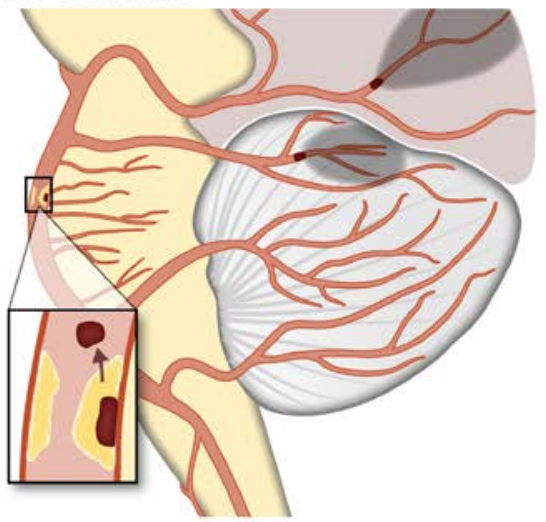

C.2

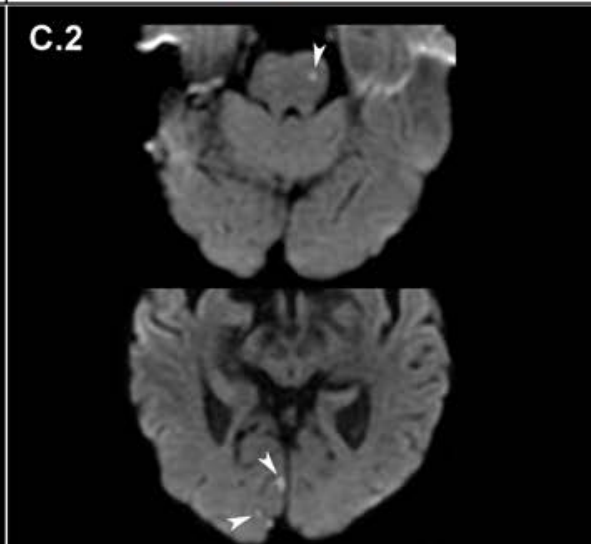

Figure 2 Stroke mechanisms of ISBAS (infarction depicted in shadows). Schematic representation in the upper row and diffusion-weighted images depicting each stroke category in the lower row. (A.1) Deep pontine stroke secondary to an unstable plaque localised in the origin of a perforator. The stroke may occur due to decreased flow (a) or in situ thrombosis (b). (A.2) Midbrain stroke (arrow) due to occlusion of a BA perforator. (B.1) Flow-dependent/hypoperfusion stroke secondary to decreased blood flow in the mid and distal BA. The atherosclerotic plaque is located in the proximal segment. (B.2) Multiple strokes affecting the cerebellum, midbrain and thalami (arrows) in the setting of a flow-dependent high-grade stenosis. (C.1) Artery-to-artery thromboembolic occlusion of distal SCA and PCA branches secondary to a plaque rupture in the mid-basilar segment. (C.2) Thromboembolic strokes (arrows) in the left midbrain and right occipital lobe. BA, basilar artery; ISBAS, isolated symptomatic basilar artery stenosis; PCA, posterior cerebral artery; SCA, superior cerebellar artery.

(mRS) score on discharge and last follow-up. For analysis purposes the mRS was dichotomised as favourable outcome (mRS 0-2) and unfavourable outcome (mRS 3-6). This study followed the Strengthening the Reporting of Observational Studies in Epidemiology guidelines. ${ }^{10}$

\section{Statistical analysis}

Discrete variables are reported as the number and percentage of patients presenting with the condition for each of the secondary outcomes. t-Test and Kruskal-Wallis test were used for continuous normal and non-normally distributed variables, respectively. $\chi^{2}$ and Fisher's exact tests were used for categorical variables. Sensitivity analysis eliminating patients presenting with acute $100 \%$ occlusion was performed in certain instances to better delineate the effect of the associations. Kaplan-Meier curves and log-rank tests were used to assess the prognostic value of collateral circulation scores, and the degree, length and location of (ISBAS) over time until recurrent TIA/ AIS or death. Events that occurred beyond 72 months of follow-up were censored for the analysis. $\mathrm{P}$ value $<0.05$ was set as statistically significant. Statistical analysis and graphic display of data were performed using GraphPad V.7.03 (GraphPad Software), SAS V.9.3 software and R V3.3.3.

\section{RESULTS}

A total of 6369 patients were screened, and 91 patients $(1.43 \%)$ met the inclusion criteria for ISBAS (figure 4). Twenty-nine $(31.9 \%)$ were women and the median age was $66.8 \pm 13.6$ years (table 1$)$. Fifty-seven $(62.6 \%$ ) patients underwent endovascular treatment, and only $4(4.3 \%)$ patients received intravenous thrombolysis. Sixty-eight (74.7\%) patients were assessed using DSA, 10 (11\%) with CTA, $9(14.3 \%)$ with MRA and $4(4.4 \%)$ with TOF. The mean follow-up time was 2.7 years. Four patients died during hospitalisation.

Female gender was associated with unfavourable outcome on discharge (OR 2.63, 95\% CI 1.03 to 6.67, $\mathrm{p}=0.04)$. Hyperlipidaemia was associated with good $\mathrm{mRS}$ score on discharge (OR 3.3, 95\% CI 1.3 to 8.1, $\mathrm{p}=0.008$ ). 


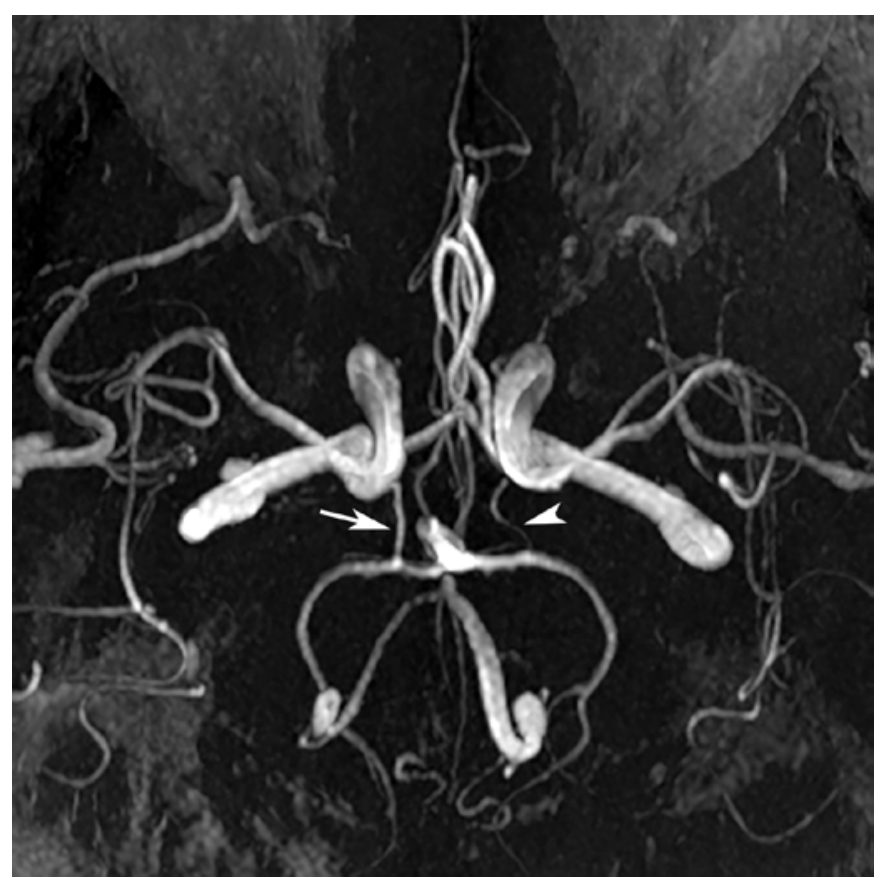

Figure 3 Axial magnetic resonance angiogram demonstrating the scoring system used to quantify the degree of collateral flow through the posterior communicating arteries (PCOM). In this case the right PCOM (arrow) is dominant (score of 2), and the left PCOM is hypoplastic (arrowhead) (score of 1). Absence of PCOM: score of 0.

However, this association was not significant for mRS score at last follow-up $(\mathrm{p}=0.27)$ or multivariable analysis, suggesting it was an incidental statistical artefact with a confounding effect. Smoking status, history of diabetes,

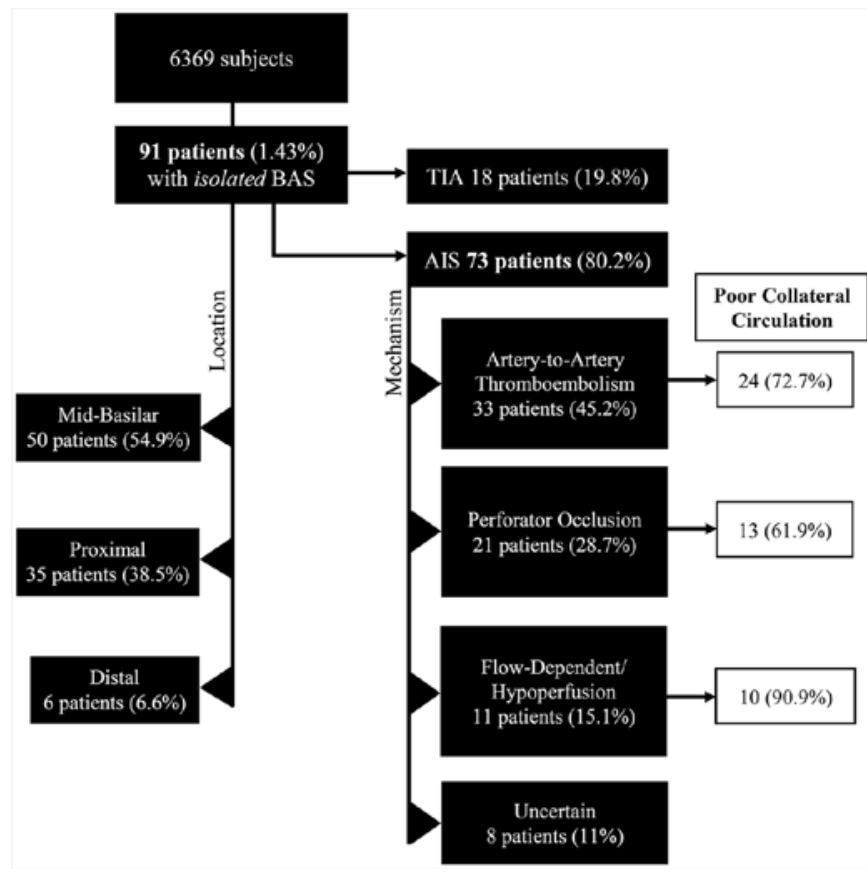

Figure 4 Flow chart of the cohort by presentation and location of the stenosis. AIS, acute ischaemic stroke; BAS, basilar artery stenosis; TIA, transient ischaemic attack.
Table 1 Baseline characteristics of the sample

\begin{tabular}{ll}
\hline Variable & Patients=91 \\
$\mathbf{n}(\%)$
\end{tabular}

NIHSS, National Institutes of Health Stroke Scale.

hypertension, coronary artery disease and previous TIA/ AIS were not associated with unfavourable outcomes.

Seventy-three $(80.2 \%)$ patients presented with AIS and $18(19.8 \%)$ with TIA. As expected, AIS presentation was significantly associated with poor outcome on discharge (OR 16, $\mathrm{p}<0.001)$. This finding was significant even in patients without complete occlusion (OR 11, $\mathrm{p}=0.006$ ). Similarly, high score on the National Institutes of Health Stroke Scale (NIHSS) at presentation was associated with mRS 3-6 at discharge (mean NIHSS score 9.0 (4.0-15.0), $\mathrm{p}<0.001)$ and at follow-up (mean NIHSS score $7.0(4.0-$ $10.0), \mathrm{p}<0.001)$.

Most patients received medical treatment with either a single antiplatelet $(12.1 \%, 11 / 91)$ or dual antiplatelets $(74.7 \%, 68 / 91)$. There was a significant association between antiplatelet therapy and good mRS score on discharge $(p<0.001)$. Inter-reader agreement was excellent for both location of BA stenosis $(\kappa=0.87,95 \% \mathrm{CI}$ 0.7 to 1.0$)$ and PCOM collateral score $(\kappa=0.92,95 \%$ CI 


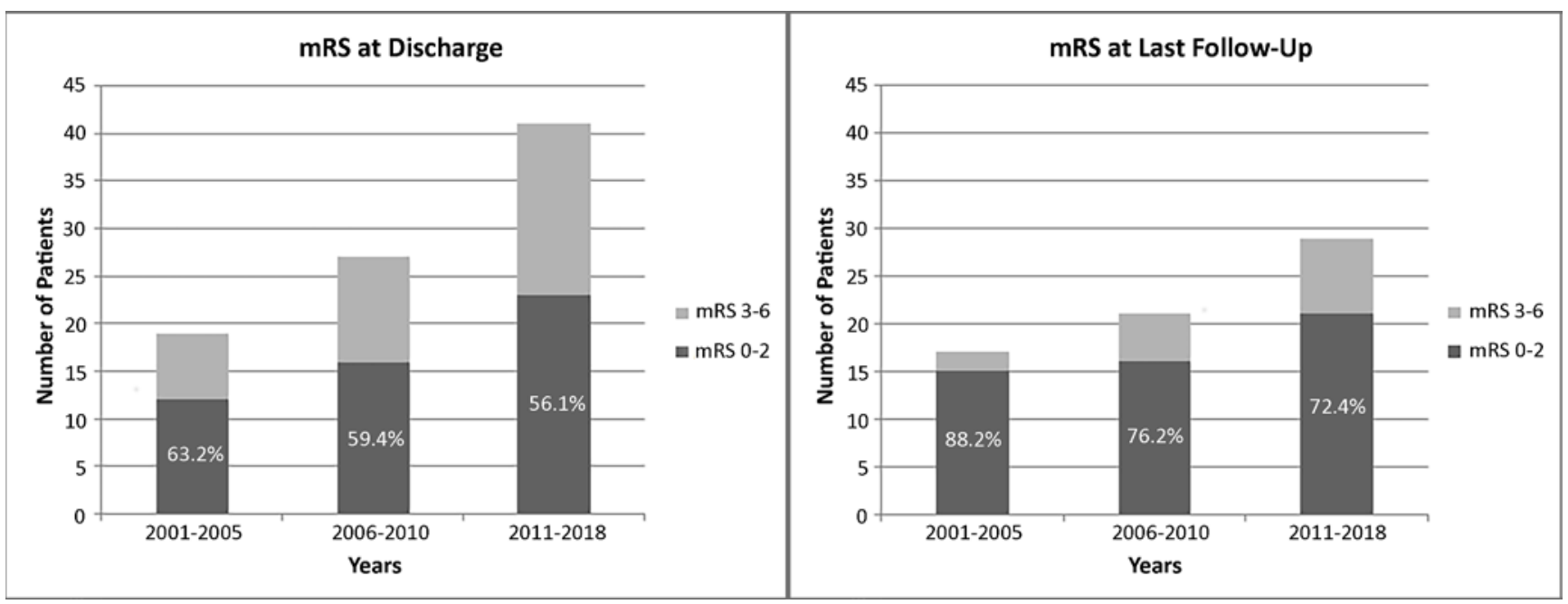

Figure 5 Bar graph depicting neurological outcomes (0-2 vs 3-6) at discharge and last follow-up. Patients enrolled from 2011 to 2015 and from 2016 to 2018 are pooled in the same time period due to a low number of patients recruited between 2016 and 2018. mRS, modified Rankin Scale.

0.87 to 0.95 ). Better neurological outcomes (mRS 0-2) at discharge and last follow-up were recorded along the 17 years of data included in the study; however, this timedependent association was not statistically significant $(p>0.05)$ (figure 5).

\section{Stroke mechanism}

In patients who presented with AIS, the stroke mechanism was artery-to-artery thromboembolism in 33 cases (45.2\%), perforator occlusion in 21 cases $(28.7 \%)$ and flow-dependent in 11 cases $(15.1 \%)$. In eight patients $(11 \%)$ the stroke mechanism was uncertain. The relationship between stroke mechanism and poor collateral circulation (score 0-1) was for flow-dependent stroke $=10 / 11$ $(90.9 \%)$, for artery-to-artery thromboembolism $=24 / 33$ $(72.7 \%)$ and for perforator=13/21 (61.9\%), without statistical significance $(\mathrm{p}=0.14)$. The stroke mechanism did not correlate with functional outcome.

\section{Degree of stenosis}

The mean percentage of stenosis was $81.8 \pm 14.7$. The percentage of stenosis was significantly lower in patients who had favourable outcomes at discharge $(78.3 \pm 14.3)$ compared with those with poor outcome $(86.9 \pm 14.5$, $\mathrm{p}=0.007$ ). When excluding patients with $100 \%$ occlusion (six mid-basilar and six proximal/distal), $\mathrm{p}$ value turned more significant between lower degree of stenosis and favourable outcome on discharge $(\mathrm{p}=0.002)$ (table 2$)$.

Most TIAs/AIS/deaths occurred during the first 6 months in Kaplan-Meier curves using $\geq 80 \%$ stenosis as cut-off value (figure $6 \mathrm{~A}$ ). When assessing for collateral circulation, 43 of $56(76.8 \%)$ patients with $<80 \%$ stenosis had no PCOMs or only one hypoplastic PCOM $($ score $=0-1)$. Conversely, 8 of $21(38.1 \%)$ patients with $\geq 80 \%$ stenosis had at least two hypoplastic or one dominant PCOMs (score=2-4) $(\mathrm{p}=0.15$; figure $6 \mathrm{~B})$. Of note, 12 $(13.2 \%)$ patients with BA occlusion were included in the study and 4 had mRS 5-6 on discharge.

\section{Location of the atherosclerotic lesion}

Fifty (54.9\%) patients had mid-basilar stenosis, 35 (38.5\%) had proximal stenosis and only $6(6.6 \%)$ had distal stenosis. Kaplan-Meier curves showed higher recurrence rates in patients with mid-basilar stenosis compared with proximal or distal stenosis. This difference was not significant in the 72-month censored curves $(\mathrm{p}=0.193)$, but statistical association increased in the 6-month censored plot $(\mathrm{p}=0.08)$ (figure 6C). There was no significant association between location of the stenosis and $\mathrm{mRS}$ score on discharge $(\mathrm{p}=0.77)$ or mRS score on last follow-up $(\mathrm{p}=0.33)$. Interestingly, patients who smoked were more likely to have mid-basilar stenosis $(\mathrm{p}=0.026)$.

\section{Length of stenosis}

The average length of stenosis was $4.7 \pm 3.0 \mathrm{~mm}$. KaplanMeier curves for length of stenosis plotted using a cut-off value of $4 \mathrm{~mm}$ showed no association with more events over time. Patients with poor outcome on discharge had a median length of stenosis of $5.2 \pm 3.8 \mathrm{~mm}$ compared with $4.3 \pm 2.4 \mathrm{~mm}$ in patients with good outcome $(\mathrm{p}=0.19)$. No significant association was found for mRS score on last follow-up $(\mathrm{p}=0.52)$.

\section{DISCUSSION}

Previous studies only included the BA in the context of diffuse vertebrobasilar atherosclerotic burden, which makes the assessment of isolated BA disease difficult. Atherosclerotic lesions of the BA are a treatment challenge due to their eloquent location and the large number of perforators that originate at this level. In this study we characterised the clinical presentation, angiographic characteristics and functional outcomes of ISBAS.

\section{Presentation}

ISBAS is uncommon. In this 17-year cohort of patients, ISBAS represented $1.4 \%$ of the total number of TIAs and 
Table 2 Univariate analysis for functional outcome at discharge and last follow-up

\begin{tabular}{|c|c|c|c|c|c|c|}
\hline \multirow[b]{2}{*}{ Variable } & \multicolumn{3}{|c|}{ mRS score at discharge } & \multicolumn{3}{|c|}{ mRS score at last follow-up } \\
\hline & $\begin{array}{l}3-6 \\
(n=36)\end{array}$ & $\begin{array}{l}0-2 \\
(n=51)\end{array}$ & P value & $\begin{array}{l}3-6 \\
(n=17)\end{array}$ & $\begin{array}{l}0-2 \\
(n=44)\end{array}$ & $P$ value \\
\hline Age & $67.3 \pm 14.4$ & $65.7 \pm 13.2$ & 0.61 & $69.8 \pm 15.1$ & $64.0 \pm 14.2$ & 0.17 \\
\hline Gender & & & 0.04 & & & 0.21 \\
\hline Female & $16(44.4)$ & $12(23.5)$ & & $7(41.2)$ & $11(25)$ & \\
\hline Male & $20(55.6)$ & 39 (76.5) & & $10(58.8)$ & $33(75)$ & \\
\hline Race & & & 0.11 & & & 0.19 \\
\hline Afro-American & $7(19.4)$ & $4(7.8)$ & & $2(11.8)$ & $1(2.3)$ & \\
\hline White/Caucasian & $29(80.6)$ & 47 (92.2) & & $15(88.2)$ & $43(97.7)$ & \\
\hline Past/current smoker & $10(27.8)$ & $17(33.3)$ & 0.58 & $3(17.6)$ & $17(38.6)$ & 0.12 \\
\hline Diabetes & 12 (33.3) & $20(39.2)$ & 0.58 & $8(47.1)$ & $14(31.8)$ & 0.27 \\
\hline Hypertension & 28 (77.8) & $43(84.3)$ & 0.44 & $14(82.4)$ & 35 (79.5) & 0.80 \\
\hline Hyperlipidaemia & $16(44.4)$ & 37 (72.5) & 0.008 & $9(52.9)$ & $30(68.2)$ & 0.27 \\
\hline Coronary artery disease & $6(16.7)$ & $14(27.5)$ & 0.24 & $2(11.8)$ & $11(25)$ & 0.26 \\
\hline $\begin{array}{l}\text { Previous stroke/transient ischaemic } \\
\text { attack }\end{array}$ & $13(36.1)$ & $21(41.2)$ & 0.63 & $8(47.1)$ & $17(38.6)$ & 0.55 \\
\hline Stroke at presentation & $35(97.2)$ & 35 (68.6) & $<0.001$ & $17(100)$ & $32(72.7)$ & 0.016 \\
\hline Stroke mechanism & & & 0.86 & & & 0.72 \\
\hline Artery-to-artery & $16(53.3)$ & $16(51.6)$ & & $9(56.3)$ & $13(48.1)$ & \\
\hline Flow-dependent & $6(20)$ & $5(16.1)$ & & $2(12.5)$ & $6(22.2)$ & \\
\hline Perforator & $8(26.7)$ & 10 (32.3) & & $5(31.3)$ & $8(29.6)$ & \\
\hline NIHSS score at presentation & $9.0[4,15]$ & $1.0(0,3)$ & $<0.001$ & $7[4,10]$ & $1.5(0,3)$ & $<0.001$ \\
\hline Percentage of stenosis & $86.9 \pm 14.5$ & $78.3 \pm 14.3$ & 0.007 & $82.6 \pm 14.4$ & $79.4 \pm 15.2$ & 0.45 \\
\hline Length of stenosis & $5.2 \pm 3.8$ & $4.3 \pm 2.4$ & 0.19 & $3.7 \pm 1.4$ & $3.8 \pm 2.4$ & 0.92 \\
\hline Location of stenosis* & & & 0.002 & & & 0.087 \\
\hline Distal & $2(5.6)$ & $4(7.8)$ & & $1(5.9)$ & $3(6.8)$ & \\
\hline Mid-basilar & $15(41.7)$ & $26(51.0)$ & & $11(64.7)$ & $20(45.5)$ & \\
\hline Proximal & $8(22.2)$ & $20(39.2)$ & & $2(11.8)$ & $18(40.9)$ & \\
\hline Collateral score & & & 0.99 & & & 0.99 \\
\hline High (2-4) & $2(6.7)$ & $4(8.9)$ & & $1(6.3)$ & $4(9.5)$ & \\
\hline Low (0-1) & $28(93.3)$ & $41(91.1)$ & & $15(93.8)$ & $38(90.5)$ & \\
\hline
\end{tabular}

Values presented as mean $\pm \mathrm{SD}$, median [P25, P75], median (minimum, maximum) or $\mathrm{n}$ (column \%).

*Excluded patients with complete basilar artery occlusion.

NIHSS, National Institutes of Health Stroke Scale;mRS, modified Rankin Scale.

AIS. Most of these patients were men $(68 \%)$, and the average age at presentation was 66 years old. Studies by Lee $e t a l$ and Kim $e t a t^{6}$ of patients (15 and 19 patients, respectively) who presented with acute BA occlusion and underlying atherosclerosis also reported an average age of 67.1 and 66.8 years old, with male predominance $(80 \%$ and $68 \%$, respectively). A case series of Bai $e t a l^{11}$ reported a mean age of 61 years old in a series of 91 patients with severe BA symptomatic stenosis; $72 \%$ were men.

As suggested by Gutierrez et $a l^{12}{ }^{12}$ vessel wall stress induced by blood flow leads to intimal thickening. These compensatory responses may regress over time. ${ }^{13}$ However, in a minority of individuals between 20 and 40 years old, arteries may undergo pathological intimal thickening. In the case of BA atherosclerosis, this process may peak and become symptomatic in the sixth decade of life. Moreover, our study showed an association between smoking and the development of isolated symptomatic mid-BA stenosis, suggesting that external risk factors may expedite BA atherogenesis. Also, a high-resolution MRI (HRMRI) study of BA stenosis $(>50 \%)$ showed that smoking was an independent risk factor of posterior circulation strokes (OR 4.4, 95\% CI 2.2 to $15.9, \mathrm{p}=0.024){ }^{14}$

\section{Angiographic characteristics}

It is unclear why some patients develop isolated BA plaque without more prominent vertebrobasilar involvement. The BA has inherent characteristics that differ from 
Percentage of stenosis

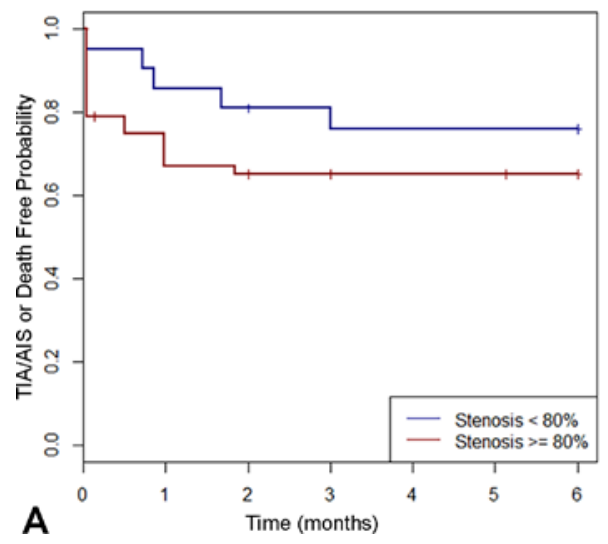

Collateral circulation score

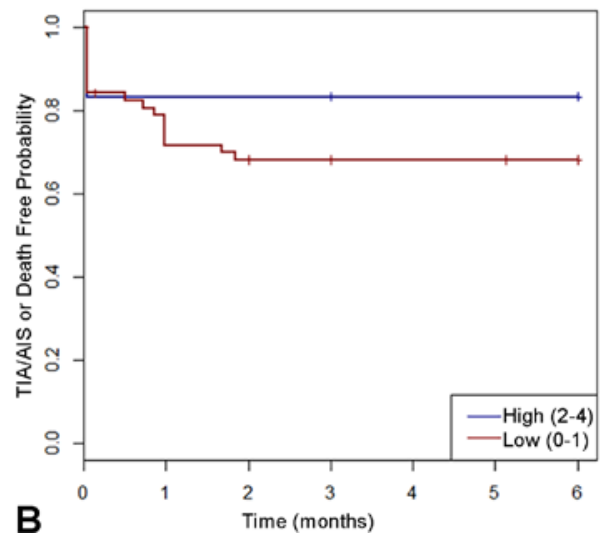

Location of stenosis

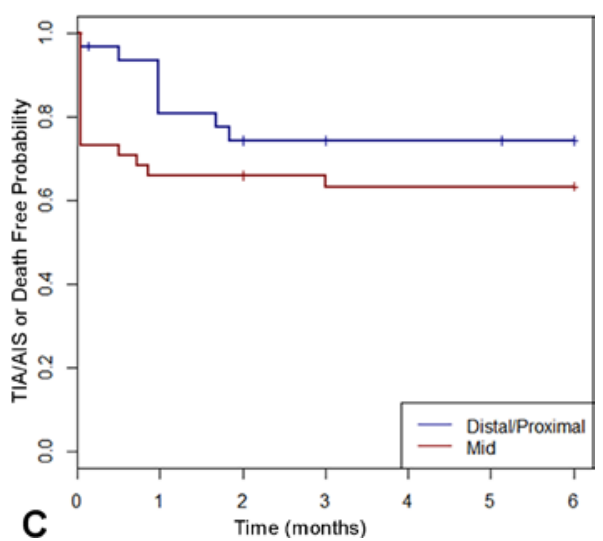

Figure 6 Kaplan-Meier curves of AIS/TIA or death at 6 months for percentage of stenosis (A), collateral circulation score (B) and location of stenosis (C). AIS, acute ischaemic stroke; TIA, transient ischaemic attack.

other arteries of the circle of Willis. Postmortem pathological studies have demonstrated that BA plaques have a concentric distribution instead of eccentric as in the anterior circulation, ${ }^{15}$ higher incidence of intraplaque haemorrhage, ${ }^{16}$ higher proportion of internal elastic lamina and less elastin as compared with the anterior circulation. ${ }^{17}$ In our cohort, mid-BA segment stenosis was the most common location correlated with higher recurrence rates in the first 6 months after the index event. This may be related to the increased number of perforators originating from the mid-BA segment. ${ }^{18}$ Kim $e t a t^{6}$ also reported that the most common location of acute BA occlusion in a cohort of patients with underlying atherosclerotic lesions was the mid-BA segment (38.5\%), compared with proximal $(30.8 \%)$ and distal locations (15.4\%). Bai et $a l^{11}$ reported similar results: mid-BA=63\%, proximal $=30.8 \%$ and distal locations $=15.4 \%$. Endovascular angioplasty and stenting of the mid-BA segment are more challenging due to the risk of disrupting the atheromatous plaque and occluding perforators with a snowploughing effect. ${ }^{190}$ A post-hoc analysis of all the complications of SAMMPRIS by Derdeyn et al demonstrated that 8 of the 10 procedural-related AIS involving the BA were primarily related to local perforator infarction, and 7 of 8 of the pontine strokes were evident immediately on awakening from anaesthesia. ${ }^{5}$ Bai et al reported a series of 91 patients who underwent endovascular BA stenting for severe symptomatic stenosis (63\% mid-BA) with a $14.3 \%$ rate of stroke and haemorrhage in the 30 -day perioperative period. ${ }^{11}$

Localisation of a distal BA occlusion is most likely embolic in nature rather than to an underlying BA atherosclerotic lesion. Distal atherosclerotic lesions of the BA are rare and should not be the first option at the time of determining the stroke mechanism of the top of the BA occlusions. Mid-BA atherosclerotic lesions more likely cause in situ thrombosis and complete BA occlusion. This difference is important at the time of endovascular intervention, as recanalisation of acute BA occlusion in this setting may entail angioplasty and even acute stenting. ${ }^{6}$ The higher incidence of mid-BA plaques as compared with proximal and distal plaques may be partially explained by haemodynamic flow turbulence at the mid-BA, where the flow bends because of the mid-BA angle. ${ }^{21}$ The blood flow recovers its laminar flow at the distal BA, which may partially explain the low incidence of distal BA stenosis $(6.6 \%)$. Length of stenosis $\geq 4 \mathrm{~mm}$ did not significantly impact the event recurrence (HR=1.14, $95 \%$ CI 0.55 to $2.37, \mathrm{p}=0.72$ ).

\section{Natural history and outcome}

The percentage of stenosis was significantly lower in patients who had favourable mRS score at discharge $(78.3 \pm 14.3)$ as compared with those with poor outcome $(86.9 \pm 14.5, \mathrm{p}=0.007)$. Collateral PCOM circulation to the top of the BA may also influence outcomes. van der Hoeven $e t a l^{22}$ showed that better collateral circulation is associated with better outcomes in BA occlusion.

Artery-to-artery thromboembolism was the most common mechanism of infarction in ISBAS. Previous studies describe in situ atherosclerotic thrombosis associated with poorer outcomes, ${ }^{4}$ while other studies used the classic Trial of Org 10172 in Acute Stroke Treatment (TOAST) classification criteria and did not find any significant relationship. ${ }^{23-26}$ Of note, these studies did not exclude patients with vertebrobasilar disease, and arteryto-artery thromboembolic events from vertebral artery atheromatous plaque played an important role in stroke mechanism. In our study, in situ thrombosis was probably the stroke mechanism in the 12 patients $(13.2 \%)$ who presented with $100 \%$ occlusion. Outcome of this population with complete BA occlusion and underlying atherosclerotic plaque is known to be poor.

To date, there is no consensus regarding treatment of patients with ISBAS who fail the maximal medical therapy used in the SAMMPRIS trial. Perhaps, there is no universal solution, and individualised approaches should be tailored to the specific vascular anatomy and pathology. A recent study showed that visualisation of BA branches is difficult with current imaging modalities, and 
HRMRI may be used to identify eccentric atherosclerotic plaques and the ostia of major BA side branches to guide endovascular therapy. ${ }^{27}$ We hypothesise that HRMRI of BA stenosis will help in selecting patients for clinical trials of endovascular treatment by better characterisation of plaque morphology, stroke mechanism and presence of perforators. Our study shows that ISBAS lesions are more common in the mid-BA segment and portend a poor prognosis with approximately $13 \%$ of patients presenting with complete occlusion.

\section{Limitations}

ISBAS is an uncommon disease in this cohort since most patients have diffuse atherosclerotic disease throughout the vertebrobasilar system. Almost $90 \%$ of patients were Caucasians, thus limiting the generalisability of our results to other Asian and non-Caucasian populations with high prevalence of intracranial atherosclerosis. This cohort of patients was screened from a 17-year long database; in this time frame intervention techniques and medical practice have changed significantly. The SAMMPRIS trial was published in 2011, and before that medical treatment of intracranial atherosclerosis was not standardised. However, this study is not aimed to determine the best treatment of ISBAS, but to reflect the natural history of the disease.

Plaque characterisation measurements were performed with different imaging modalities (DSA, CTA, MRA and TOF), which decreases accuracy. However, the percentage of stenosis should not be affected by this heterogeneity. Furthermore, conventional MRA/TOF can underestimate the degree of stenosis due to the presence of an atherosclerotic plaque. Cross-sectional HRMRI has shown that $15 \%$ of patients who initially had irregular or normal BA on MRA indeed had $\geq 40 \%$ stenosis. ${ }^{28}$ Therefore, the incidence of ISBAS may be underestimated with current imaging modalities. Finally, stroke mechanisms cannot be determined with complete certainty.

\section{CONCLUSION}

ISBAS represents about $1.5 \%$ of AIS and TIAs. It is more common in men, mostly in the mid-60s. The mid-BA segment is most commonly affected, and the most common stroke mechanism is artery-to-artery thromboembolism. Patients with mid-BA stenosis, $\geq 80 \%$ stenosis and poor collateral circulation have worse functional outcomes than those with proximal or distal locations, lesser degree of stenosis and at least one dominant PCOM. Approximately $13 \%$ of these patients present with a complete BA occlusion.

Contributors EAS, AS and EL: conception and design of the study. EAS, AS, JAR, $\mathrm{BD}$ : acquisition and analysis of data. EAS, JAR, EL: drafting of the manuscript/ preparation of figures. SO-G, DMH, CD, HA: substantial scientific revision.

Funding The authors have not declared a specific grant for this research from any funding agency in the public, commercial or not-for-profit sectors.

Competing interests None declared.

Patient consent for publication Not required.

Provenance and peer review Not commissioned; externally peer reviewed.
Data availability statement Data are available upon reasonable request.

Open access This is an open access article distributed in accordance with the Creative Commons Attribution Non Commercial (CC BY-NC 4.0) license, which permits others to distribute, remix, adapt, build upon this work non-commercially, and license their derivative works on different terms, provided the original work is properly cited, appropriate credit is given, any changes made indicated, and the use is non-commercial. See: http://creativecommons.org/licenses/by-nc/4.0/.

ORCID iD

Jorge A Roa http://orcid.org/0000-0003-4987-8853

\section{REFERENCES}

1. Lee JS, Hong JM, Lee KS, et al. Endovascular therapy of cerebral arterial occlusions: intracranial atherosclerosis versus embolism. $J$ Stroke Cerebrovasc Dis 2015;24:2074-80.

2. Caplan LR, Wityk RJ, Glass TA, et al. New England medical center posterior circulation registry. Ann Neurol 2004;56:389-98.

3. Strbian D, Sairanen T, Silvennoinen $\mathrm{H}$, et al. Intravenous thrombolysis of basilar artery occlusion: thrombus length versus recanalization success. Stroke 2014;45:1733-8.

4. Lee W-J, Jung K-H, Ryu YJ, et al. Impact of stroke mechanism in acute basilar occlusion with reperfusion therapy. Ann Clin Transl Neurol 2018;5:357-68.

5. Derdeyn CP, Fiorella D, Lynn MJ, et al. Mechanisms of stroke after intracranial angioplasty and stenting in the SAMMPRIS trial. Neurosurgery 2013;72:777-95. discussion 95.

6. Kim YW, Hong JM, Park DG, et al. Effect of intracranial atherosclerotic disease on endovascular treatment for patients with acute vertebrobasilar occlusion. AJNR Am J Neuroradiol 2016;37:2072-8.

7. Samuels OB, Joseph GJ, Lynn MJ, et al. A standardized method for measuring intracranial arterial stenosis. AJNR Am J Neuroradiol 2000;21:643-6.

8. Derdeyn CP. Mechanisms of ischemic stroke secondary to large artery atherosclerotic disease. Neuroimaging Clin N Am 2007;17:303-11. vii-viii.

9. Sussman ES, Kellner CP, Mergeche JL, et al. Radiographic absence of the posterior communicating arteries and the prediction of cognitive dysfunction after carotid endarterectomy. J Neurosurg 2014;121:593-8.

10. von Elm E, Altman DG, Egger M, et al. Strengthening the reporting of observational studies in epidemiology (STROBE) statement: guidelines for reporting observational studies. BMJ 2007;335:806-8.

11. Bai W-X, Gao B-L, Li T-X, et al. Wingspan stenting can effectively prevent long-term strokes for patients with severe symptomatic atherosclerotic basilar stenosis. Interv Neuroradiol 2016;22:318-24.

12. Gutierrez J, Elkind MSV, Virmani R, et al. A pathological perspective on the natural history of cerebral atherosclerosis. Int J Stroke 2015;10:1074-80.

13. Stary $\mathrm{HC}$, Blankenhorn $\mathrm{DH}$, Chandler $\mathrm{AB}$, et al. A definition of the intima of human arteries and of its atherosclerosis-prone regions. A report from the Committee on vascular lesions of the Council on arteriosclerosis, American heart association. Arterioscler Thromb 1992;12:120-34.

14. Wang W, Yang Q, Li D, et al. Incremental Value of Plaque Enhancement in Patients with Moderate or Severe Basilar Artery Stenosis: 3.0T High-Resolution Magnetic Resonance Study. Biomed Res Int 2017;2017:4281629.

15. Yang WJ, Fisher M, Zheng L, et al. Histological characteristics of intracranial atherosclerosis in a Chinese population: a postmortem study. Front Neurol 2017;8.

16. $\mathrm{Yu} \mathrm{JH}, \mathrm{Kwak} \mathrm{HS}$, Chung GH, et al. Association of intraplaque hemorrhage and acute infarction in patients with basilar artery plaque. Stroke 2015;46:2768-72.

17. Roth W, Morgello S, Goldman J, et al. Histopathological differences between the anterior and posterior brain arteries as a function of aging. Stroke 2017;48:638-44.

18. Marinković SV, Gibo H. The surgical anatomy of the perforating branches of the basilar artery. Neurosurgery 1993;33:80-7.

19. Levy El, Howington JU, Engh JA, et al. Submaximal angioplasty and staged stenting for severe posterior circulation intracranial stenosis: a technique in evolution. Neurocrit Care 2005;2:189-97.

20. Samaniego EA, Hetzel S, Thirunarayanan S, et al. Outcome of symptomatic intracranial atherosclerotic disease. Stroke 2009;40:2983-7.

21. Kim BJ, Lee KM, Kim HY, et al. Basilar artery plaque and pontine infarction location and vascular geometry. $J$ Stroke 2018;20:92-8.

22. van der Hoeven EJ, McVerry F, Vos JA, et al. Collateral flow predicts outcome after basilar artery occlusion: the posterior circulation collateral score. Int J Stroke 2016;11:768-75. 
23. Jung S, Mono M-L, Fischer U, et al. Three-Month and long-term outcomes and their predictors in acute basilar artery occlusion treated with intra-arterial thrombolysis. Stroke 2011;42:1946-51.

24. Strbian D, Sairanen T, Silvennoinen $\mathrm{H}$, et al. Thrombolysis of basilar artery occlusion: impact of baseline ischemia and time. Ann Neurol 2013;73:688-94.

25. Singer OC, Berkefeld J, Nolte $\mathrm{CH}$, et al. Mechanical recanalization in basilar artery occlusion: the ENDOSTROKE study. Ann Neurol 2015;77:415-24.
26. Lee YY, Yoon W, Kim SK, et al. Acute basilar artery occlusion: differences in characteristics and outcomes after endovascular therapy between patients with and without underlying severe atherosclerotic stenosis. AJNR Am J Neuroradiol 2017;38:1600-4.

27. Jiang $\mathrm{W}-\mathrm{J}, \mathrm{Yu} \mathrm{W}, \mathrm{Ma} \mathrm{N}$, et al. High resolution $\mathrm{MRI}$ guided endovascular intervention of basilar artery disease. J Neurointerv Surg 2011;3:375-8.

28. Klein IF, Lavallée PC, Mazighi M, et al. Basilar artery atherosclerotic plaques in paramedian and lacunar pontine infarctions: a highresolution MRI study. Stroke 2010;41:1405-9. 